\title{
La construcción del conocimiento sobre la enseñanza desde la perspectiva de los futuros docentes ${ }^{1}$
}

\section{The construction of knowledge about teaching from the prospective teachers' perspective}

\author{
María A. Chacón-Corzo ${ }^{2}$ \\ Universidad de Los Andes Dr. Pedro Rincón Gutiérrez \\ Táchira - Venezuela \\ corzomar@gmail.com
}

Recibido: 2 junio 2013 Aceptado: 4 diciembre 2014 Corregido: 26 noviembre 2014

Resumen: Este trabajo tuvo como objetivo indagar sobre la construcción del conocimiento sobre la enseñanza de los futuros docentes y caracterizarlo con base en sus percepciones. Es un estudio cualitativo que pretende comprender e interpretar los significados y creencias acerca del conocimiento docente. Participaron diecinueve cursantes del último semestre de la carrera de Educación Básica Integral de la Universidad de Los Andes Dr. Pedro Rincón Gutiérrez en el estado Táchira-Venezuela. Las entrevistas y diarios reflexivos de los pasantes fueron la técnica e instrumento para recolectar la información. Los datos se analizaron con el programa Atlas-ti 6. Producto del análisis emergieron las categorías: Conocimiento pedagógico, desarrollo de la competencia reflexiva y experiencias formativas; éstas explican las dimensiones acerca del conocimiento docente de los futuros profesores. En conclusión, los participantes asumen el conocimiento pedagógico a partir de la organización y gestión de la clase; consideran la planificación y la evaluación como procesos inherentes a la actividad del docente; otorgan especial interés a las estrategias de enseñanza para potenciar los aprendizajes y preponderan las prácticas profesionales como un ámbito importante, aunque declaran que durante el trayecto formativo tuvieron escasas oportunidades de interactuar en los contextos escolares. En síntesis, la construcción del conocimiento docente es un proceso complejo y multidimensional derivado de variadas fuentes; exige procesos reflexivos que den cuenta de sus progresos y limitaciones, de manera que los futuros docentes alcancen mayores niveles de reflexión y asuman el aprendizaje permanente como una condición de la carrera docente ${ }^{3}$.

Palabras clave: Formación de profesores, educación básica, aprendizaje, conocimiento y enseñanza.

Abstract: The objective of this paper was to explore and characterize the construction of knowledge based on the perceptions of prospective teachers. It is a qualitative study that attempts to understand and interpret the meanings and beliefs about teacher knowledge. The participants were the last semester students enrolled in Basic Integral Education at the University of Los Andes Dr. Pedro

1 La autora agradece el financiamiento otorgado por el CDCHTA de la Universidad de Los Andes al proyecto de investigación titulado "La construcción del conocimiento sobre la enseñanza en la formación inicial: Configuraciones y Prospectiva". NUTA-H-30109-04-B, cuyos resultados parciales se presentan en este artículo.

2 Doctora por la Universidad Rovira i Virgili, Tarragona - España. Trabaja como profesora Asociada de la Universidad de Los Andes Dr. Pedro Rincón Gutiérrez Estado Táchira, Venezuela. Ha presentado ponencias nacionales e internacionales. Entre las publicaciones recientes se encuentran: El Uso del Portafolio en la Enseñanza de Lenguas Extranjeras. Los proyectos de aprendizaje interdisciplinarios en la formación docente. Correo electrónico: mariach@ula.ve

3 También, se sugiere consultar trabajos anteriores acerca de los procesos reflexivos durante la formación, tales como "Las estrategias de enseñanza reflexiva en la formación inicial docente” cuya fuente aparece registrada en las referencias bibliográficas. 
Rincón Gutiérrez in Táchira state-Venezuela. The techniques used to collect data were the interview and reflective diaries written by the participants during their field experiences. The software Atlas-ti version 6.0 was used to analyze data. As a result of the analysis the following categories emerged: Pedagogical knowledge, development of reflection as a competence, and formative experiences. These findings explain the dimensions about teacher knowledge from the prospective teachers' perspective. It is a complex and multidimensional process that comes from several sources and demands reflective processes that tell about their progress and limitations, so that prospective teachers reach higher levels of reflection that contribute to understand teaching as a task that demands disposition for long life learning.

Key words: teacher development, Basic Education, learning, knowledge, teaching.

\section{Introducción}

La formación inicial es una de las etapas más importantes, en tanto favorece el desarrollo de una serie de competencias, habilidades, destrezas, valores, actitudes, entre otros, que acreditan al estudiante de educación para asumir su rol como profesor. Para algunos autores como Montero (2002) se trata de un período relativamente corto e insuficiente en el cual los futuros profesores alcanzan unas competencias mínimas para enfrentar la tarea de enseñar. Es decir, es el comienzo de la carrera docente, por consiguiente, sólo es una etapa inicial que proporciona los cimientos fundamentales para adentrarse en la formación continua y del desarrollo profesional; esto sin obviar su preponderancia como un ciclo crucial porque es el eje estructurador del desarrollo profesional.

En tal sentido, la trascendencia de la formación del educador, su perfeccionamiento, desarrollo y crecimiento trasciende los ámbitos formales y de prosecución de la carrera docente e implica el compromiso y la aceptación de ser aprendices permanentes de la enseñanza. Vaillant y Marcelo (2001) expresan que "se insiste, particularmente, en fomentar en los profesores actitudes de apertura, reflexividad, tolerancia, aceptación de las diferencias individuales y grupales" (p. 42). En este orden de ideas, la tarea de las instituciones encargadas de la formación debe estar orientada a promover y consolidar actitudes favorables hacia la reflexión crítica, la investigación y la producción de conocimientos sobre los procesos educativos.

De esta manera cobra fuerza la idea de cooperar en la apropiación de saberes y competencias docentes acordes con las exigencias de la sociedad actual. Por tal razón, es importante indagar sobre cómo construye y reconstruye su conocimiento el futuro docente; ello hace inevitable profundizar en las concepciones y teorías implícitas que subyacen en la tarea de enseñar. Vale resaltar que estos saberes previos se hacen evidentes cuando los estudiantes se enfrentan durante las Prácticas Profesionales a los contextos escolarizados y asumen los roles del educador. Tal como lo expresa Davini (2001):

Como producto de la trayectoria anterior a su ingreso a las instituciones de formación inicial, a través de su experiencia como alumnos. Como productos internalizados a lo largo de su historia escolar, este 'fondo de saber' orientaría en buena medida las formas de asumir su propio papel como docentes. (p. 80) 
Es decir, la historia personal se hace presente mediante las creencias acerca de la tarea de enseñar, por ello es necesario, durante la formación, promover la reflexión acerca de los saberes y experiencias que pudieran influir en la actuación como profesores. Por su parte, Bromberg, Kirsanov y Longueria (2008) afirman que tanto los docentes como los futuros docentes saben sobre la escuela; lo saben porque lo han aprendido, precisamente, en el entorno escolar, "pero en una posición distinta, la de alumno" (p. 15). Por tanto, es fundamental explicitar la cosmovisión que poseen los futuros educadores; al respecto, Martín y Cervi (2006) afirman la conveniencia de descubrir las concepciones que subyacen en las acciones de los aprendices como un modo de reflexionar y transformar su pensamiento y acción.

Por otra parte, hoy en día los conocimientos que puede poseer el docente le capacitan o no para enfrentar los retos de la cotidianidad del aula y el contexto escolar, impregnado de incertidumbre y relatividad. En otras palabras, no es suficiente conocer y dominar la técnica, los procedimientos y los medios para asumir la tarea de enseñar. Es necesario estar consciente de la complejidad de la acción educativa y poseer la capacidad para tomar decisiones en la práctica propiamente dicha. Es decir, no se puede normatizar o tecnificar la enseñanza ni los procedimientos a seguir porque ello reduciría los conocimientos del educador a un enfoque meramente técnico. Por el contrario, tal como afirman Diker y Terigi (2003) se trata de "definir dispositivos a través de los cuales sea posible explicitar el conocimiento de los docentes y convertirlo en experiencias susceptibles de análisis personal, y comunicables a otros, trátese de colegas en cursos de perfeccionamiento o de docentes en formación" (p. 109).

En otras palabras, abordar la acción comunicativa, la actuación docente y revelar los supuestos que fundamentan estas actuaciones, para reorientarlas o enriquecerlas, contribuye a hacer consciente la acción y cómo se aprende a enseñar. Después de todo, es un proceso rodeado de complejidad y pluridimensional, dado que se trata de conocimientos diversos, aprendidos en diferentes ámbitos y momentos desde la trayectoria escolar previa (desde la educación primaria hasta la formación universitaria) y la socialización a través de las prácticas profesionales.

En ese sentido, las prácticas profesionales pueden convertirse en ámbitos potenciadores para la construcción del conocimiento sobre la enseñanza. Siguiendo a Korthagen (2010), los estudiantes reflexionan con base en sus propias experiencias. En tal sentido, los formadores podrían ubicarse en un enfoque de formación realista que establezca un continuum entre teoría y práctica. La vivencia de situaciones reales que deriven en preocupaciones puede incitar a la reflexión tanto individual como grupal a fin de construir y reconstruir el conocimiento. Korthagen (2010) asevera que "Si queremos que las escuelas se conviertan en comunidades de práctica, con educadores que desarrollen juntos su propia habilidad, debemos ayudarles a acostumbrarse a formas de aprendizaje cooperativo y colaborativo durante el procesos de formación del profesorado" (p. 87).

En este estudio, se suscriben los planteamientos de este autor acerca de la necesidad de impulsar la reflexión crítica entre pares. Deliberar y confrontar las teorías subyacentes en las acciones docentes contribuye a "tender puentes entre teoría y práctica" (p.92). Este proceso posibilita que los futuros profesores concienticen lo que sucede con su propio pensamiento en relación con la enseñanza y el aprendizaje en los contextos escolares.

Obviamente, debe tenerse presente la multiplicidad de agentes y elementos que intervienen y conviven en los espacios educativos que obligan a mirar la trascendencia de 
un objeto de estudio dinámico, flexible y en constante reconstrucción, dada la convergencia de diversas disciplinas que se articulan para dar sentido al ejercicio profesional del docente. Sayago y Chacón (2006) señalan que en las prácticas influyen las concepciones de enseñanza, aprendizaje, tradiciones de formación docente y características propias del contexto socio cultural; es decir, surgen multiplicidad de posibilidades para que el futuro docente pueda aprender a analizar críticamente su actuación de aprendiz y de enseñante, siempre que alcance a discutir, contrastar y reconstruir la experiencia docente, esto es, un camino para construir su conocimiento profesional.

Ahora bien, sobre conocimiento profesional docente se han generado innumerables reflexiones que lo señalan como aquellos saberes que el docente pone en acción durante la enseñanza. Como ya se ha afirmado, se trata de conocimientos diversos, complejos y multireferenciales; así se asevera que este conocimiento no es únicamente teórico sino que es complementario y simultáneamente práctico. No obstante, no se trata de validar la teoría, sino que teoría y práctica interactúan y se complementan para conformar el conocimiento sobre la profesión; apoyado en las concepciones sobre enseñanza, aprendizaje y todas aquellas experiencias experimentadas durante el trayecto profesional.

Badía y Monereo (2004) conciben el conocimiento profesional docente como representaciones cognitivas orientadas a la práctica; éstas ayudan a la interpretación de los problemas de enseñanza y aprendizaje que debe afrontar el docente y que orientan el rumbo de las actividades de enseñanza, aprendizaje y evaluación en el ejercicio profesional. Por su parte, Porlán, Rivero y Martín (1997) afirman que éste es un conocimiento práctico por cuanto la naturaleza de los problemas está vinculada a la enseñanza como una actividad práctica.

También es conveniente señalar que diversos autores han establecido categorías del conocimiento. En este estudio, se refieren las propuestas por Shulman (2005): (a) conocimiento de los alumnos, (b) conocimiento didáctico general, (c) conocimiento del contenido pedagógico, (d) conocimiento del contenido, (e) conocimiento del currículo, (f) conocimiento de los objetivos, las finalidades, los valores y fundamentos filosóficos e históricos y (g) conocimiento del contexto educativo.

Según este autor estas categorías corresponden a aquellos saberes sobre los cuales se sustenta el conocimiento base de la enseñanza. En cuanto a la naturaleza del conocimiento, el mismo Shulman expresa que éste procede de diversas fuentes como la formación académica de la disciplina a enseñar, materiales y contexto del proceso educativo institucionalizado, textos, organización escolar, estructura de la profesión; los estudios sobre la escuela, organizaciones sociales, aprendizaje humano, enseñanza, desarrollo y otros ámbitos socioculturales que influyen en la acción docente. Finalmente, añade a la sabiduría como otro principio que proporciona saberes en la práctica tanto del aula como de la institución escolar.

Dada su naturaleza y complejidad es importante aclarar que, para este trabajo, el conocimiento docente se asume como la apropiación de conceptos, procedimientos y actitudes que, transferidos a la práctica, coadyuvan en la interpretación y resolución de situaciones de enseñanza y aprendizaje a las que se enfrenta el profesor.

Por otra parte, es preciso señalar que este estudio se propuso indagar sobre los modos cómo los estudiantes de la carrera de Educación Básica Integral (educación primaria) construyen el conocimiento sobre la enseñanza, sin obviar que se trata de sujetos con una 
historia personal imbricada por sus saberes, experiencias y un contexto sociocultural en el cual han vivido. Sin duda, estas vivencias les hacen acreedores de una cultura y aprendizaje escolar que inciden en sus creencias sobre cómo enseñar. Al respecto Tardif (2004) expresa que "los docentes utilizan en sus actividades cotidianas conocimientos prácticos que provienen del mundo vivido, de los saberes, de sentido común, de las competencias sociales" (p.100).Por su parte Elliot (1999) expresa que:

Se considera de especial relevancia la experiencia previa que, como estudiantes, han acumulado a lo largo de años en las aulas, de forma que se produciría, por esta vía, una interiorización de los modelos de enseñanza que sus profesores practicaron con ellos. (p. 382)

Por ello nos interesa reconocer al sujeto que aprende y desaprende con base en sus experiencias formativas y también aquellas que le ofrece el trayecto de formación inicial. En tal sentido, este estudio tuvo como intención indagar sobre la construcción del conocimiento de los futuros profesores, cursantes de la carrera de Educación Básica Integral (EBI) de la Universidad de Los Andes Táchira "Dr. Pedro Rincón Gutiérrez". Por tanto, el proceso de investigación parte de las siguientes interrogantes: ¿Cuáles son los tipos de conocimientos que los futuros profesores creen que deben construir o aprender para enseñar? ¿Reconocen los estudiantes de educación, de manera consciente, el aprendizaje de la enseñanza como un proceso permanente en el ejercicio docente?

Con base en estas cuestiones, los objetivos se orientaron hacia: Indagar acerca del conocimiento que construyen los futuros profesores sobre la enseñanza y caracterizar las dimensiones que consideran fundamentales para el ejercicio profesional.

\section{Método}

Es un estudio cualitativo desde la perspectiva interpretativa porque interesa comprender los significados y creencias de los estudiantes practicantes acerca del conocimiento docente. Se utilizó la entrevista como técnica para profundizar en las concepciones y puntos de vista de los estudiantes, y los diarios reflexivos por cuanto permitían comprender las ideas, vivencias y perspectivas sobre aprender a enseñar de cada participante.

De la entrevista puede señalarse que se trató de conversaciones con los participantes para profundizar sobre sus vivencias y aprendizajes durante el proceso de prácticas. Posteriormente, se entregó la entrevista transcrita a cada uno para que corroborara sus opiniones. En relación con los diarios, se motivó a los practicantes a escribir sobre sus experiencias, las actividades docentes que planificaban y llevaban a cabo. Asimismo, se les invitó a escribir sobre sus aprendizajes, inquietudes, los imprevistos que se presentaban y las acciones que emprendían para resolverlos. Cada uno elaboró al menos tres diarios, los cuales fueron leídos y releídos por la investigadora a fin de encontrar elementos comunes. Luego se procedió a la codificación, interpretación y síntesis de la información, lo que derivó en el establecimiento de las categorías y subcategorías. La información fue analizada mediante el programa Atlas-ti 6, a fin de establecer conceptos y relaciones con base en los datos (Coffey y Atkinson, 2003) los cuales son aportes importantes para comprender el fenómeno en estudio. 
Tal como se ha señalado, el estudio se realizó en la Universidad de Los Andes en el estado Táchira, con diecinueve estudiantes cursantes de la carrera de Educación Básica Integral, que desarrollaban su práctica docente en instituciones de educación primaria en la ciudad de San Cristóbal, Venezuela. Los criterios para seleccionar a los participantes fueron: (a) manifestaron interés por la investigación, (b) cursantes del último semestre de la carrera de educación y (c) con escasa o ninguna experiencia anterior como docentes.

Es importante señalar que, a fin de ilustrar la interpretación en cada categoría y subcategoría, se ejemplifica con algunos fragmentos en cursiva de los documentos primarios que contienen los testimonios de los participantes. Estos identifican al participante con una letra mayúscula y un número, seguido del número de línea del documento, por ejemplo: [P12, 61].

\section{Hallazgos}

Se procedió a analizar, codificar y organizar los datos con el fin de llegar a teorizar sobre la construcción del conocimiento docente (Strauss y Corbin, 2002). A partir de este análisis en profundidad, surgen las categorías Conocimiento Pedagógico, Desarrollo de la Competencia Reflexiva y Experiencias Formativas, que serán descritas a continuación.

\section{Categoría: Conocimiento pedagógico}

Se asume como todas aquellas dimensiones que, según la percepción de los participantes en el estudio, se constituyen en saberes, conocimientos y experiencias que han posibilitado la construcción de aprendizajes en estos futuros educadores. Comprende los ámbitos aprendidos y desarrollados durante el trayecto de formación y que, de algún modo, se concretan en la práctica docente, es decir, los estudiantes aprecian esta construcción fuertemente vinculada a sus prácticas. Asimismo, surgen las subcategorías: Gestión de la clase, estrategias de enseñanza y contexto como soportes que influyen en la construcción de este conocimiento (ver Figura 1).

En relación con la subcategoría Gestión de la Clase, emergen dimensiones vinculadas a la planificación, la evaluación, los contenidos, los recursos, medios y materiales. En cuanto a la planificación, comprende el proceso que implica diseñar y elaborar los proyectos de aula para los cuales se requiere de un conocimiento previo; caben aquí las concepciones que se tienen sobre planificación, enseñanza, estrategias, entre otros.

En el siguiente fragmento se puede apreciar la opinión de uno de los participantes: hay que tener en cuenta una serie de aspectos para llevar a cabo un plan, debidamente organizado para obtener los objetivos propuestos en el Proyecto de Aprendizaje. Por lo tanto, hay que prepararse, leer e investigar cada tema que se va a desarrollar con los niños, para evitar, la improvisación y reducir la incertidumbre para saber abordar posibles preguntas que puedan hacer los niños, coordinando así la participación de todos y garantizando el uso racional y acorde del tiempo disponible para cada actividad [P13, 137:145].

De acuerdo con el testimonio anterior, se evidencia que los participantes reconocen la necesidad de prepararse y prever una serie de procedimientos para organizar y elaborar los 
proyectos de aprendizaje. Entre éstos, destacan las intencionalidades educativas, es decir explicitar los objetivos educativos que se aspira lograr. Otro aspecto considerado fundamental es la observación porque contribuye a explorar las necesidades, intereses y caracterizar los grupos de clase. Igualmente, es esencial indagar acerca de los temas a estudiar, las estrategias y recursos requeridos para concretar las intenciones educativas planteadas. También, se manifiesta la preocupación por aprovechar los lapsos disponibles para desarrollar los proyectos; lo cual podría interpretarse como una valoración del tiempo en el aula.

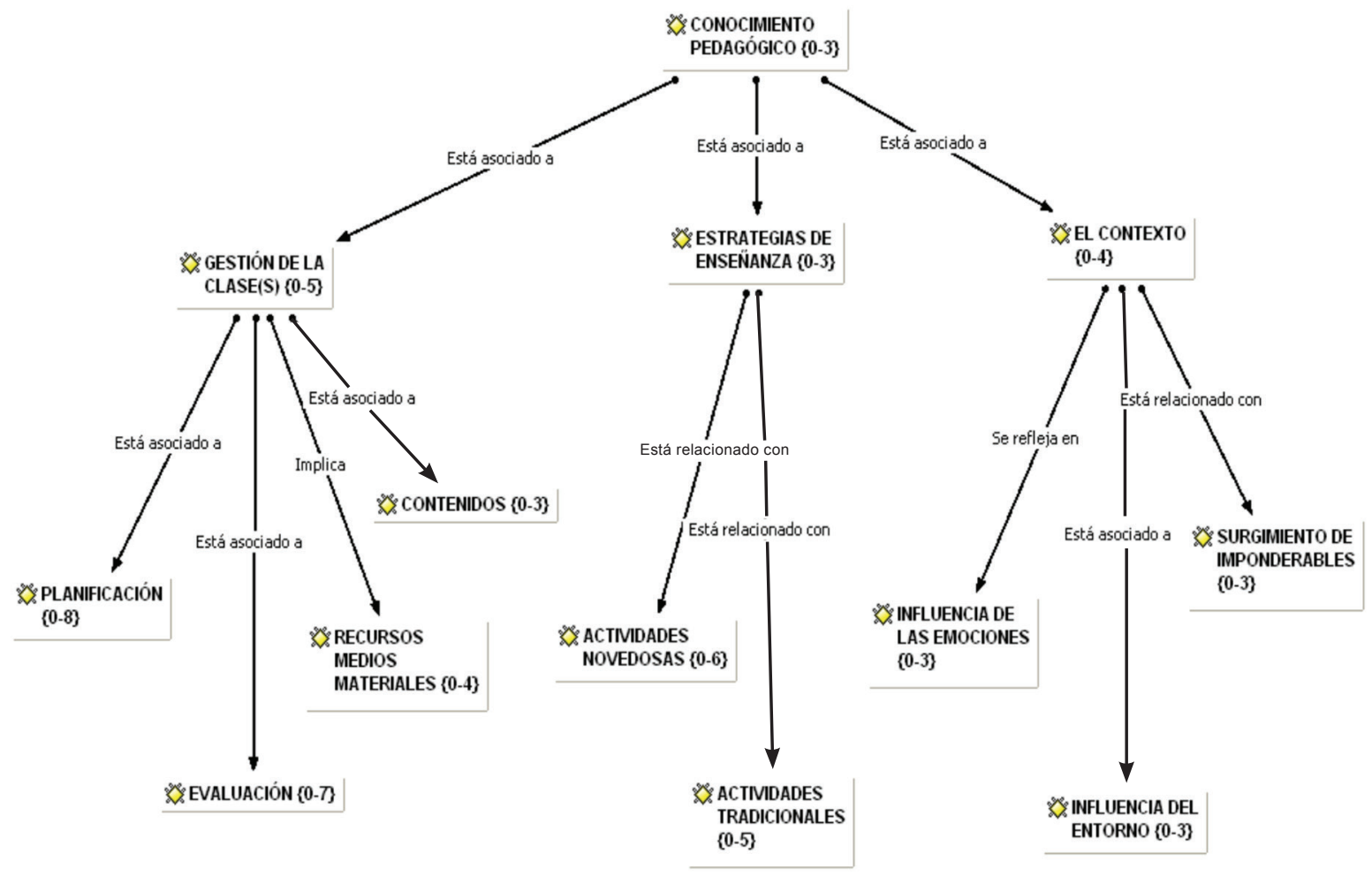

Figura 1: Conocimiento pedagógico.

Nota: Proceso de investigación

En la evaluación prevalece el conocimiento de los tipos de evaluación: formativa, sumativa y de diagnóstico, los momentos de evaluación y por supuesto, las técnicas e instrumentos de evaluación. No obstante, reconocen debilidades para llevar a cabo la evaluación cualitativa que corresponde a la educación primaria. Por otra parte, tenemos los contenidos, donde todos los participantes coinciden en la importancia de dominar los contenidos a enseñar. $\mathrm{Al}$ respecto, se reafirma la necesidad de conocer a los alumnos y el contexto, de modo que el aprendizaje de los conceptos, procesos y actitudes se contextualicen a los requerimientos de los grupos de clase con los que trabajaron los futuros docentes. Así se refleja en los siguientes comentarios: Conocer las áreas curriculares de la educación primaria, la relación interdisciplinar entre ellas y los criterios de evaluación [P10, 170:171]. El maestro tiene que saber que va a explicar o mejor dicho debe conocer el tema que va a explicar para su aprendizaje [P4, 13:15]. 
En cuanto a la dimensión relativa a los recursos, medios y materiales, prevalece el apoyo en material concreto, libros de textos como enciclopedias, algunos cuentos y leyendas, entre otros. Se percibe el carácter instrumental de los materiales utilizados para obtener información, más que un medio para abordar críticamente la realidad. Asimismo, consultan el Curriculum Oficial para concretar los contenidos y seleccionar los recursos para la enseñanza. Evidencia de ello son los siguientes extractos: se usaron las enciclopedias Girasol, Tricolor, Didácticas porque son textos de referencia en la enseñanza de varios contenidos y es muy usado en el entorno escolar, además de los buscadores de internet para obtener información actualizada y completar las estrategias. [P1, 168:171]. Estas fueron planificadas y utilizadas en base a los propósitos y contenidos de los proyectos de aprendizaje, consultando el Currículo Básico Nacional [P17, 32:34].

En relación con la subcategoría Estrategias de enseñanza (ver Figura 1), ésta se refiere a los procedimientos, las acciones y los recursos, utilizados por los pasantes, que han constituido una fuente de construcción del conocimiento; comprende actividades novedosas caracterizadas por el trabajo en equipo y de campo, lecturas y en menor grado, la promoción de la escritura. Seguidamente, algunos ejemplos: La recreación de los alumnos a través de la lectura como instrumento fundamental de su aprendizaje, ya que para muchos de ellos leer se les hace aburrido, y exitosamente mi entrada al aula los ha motivado significativamente [P15, 182:185].

También corresponden a esta subcategoría las actividades tradicionales en las que se encuentran dictados, caligrafías, entre otros. El siguiente testimonio es ilustrativo: Primero les dictaba lo más especifico del tema y luego elegía explicarles detalladamente lo que se estaba trabajando [P3, 107:108].

En los testimonios anteriores subyacen las concepciones sobre enseñanza de los participantes en el estudio, por una parte, una visión de la enseñanza y el aprendizaje como construcción de conocimiento y, por otra, la visión tradicional limitada al dictado y la explicación del profesor. Al respecto, se hace necesario señalar que persisten las concepciones en razón de las experiencias vividas. Por tanto, se hace necesario, tal y como lo expresan Cruz, Pozo, Huarte y Scheuer (2006), explicitar y confrontar las concepciones de los futuros profesores a fin de deconstruir y reconstruir el conocimiento sobre la enseñanza con miras a formar docentes reflexivos.

La subcategoría el contexto (Figura 1) comprende el entorno, surgimiento de imponderables e influencia de la emociones. El entorno vinculado al reconocimiento de intereses y necesidades así como la convivencia escolar son factores que inciden en la enseñanza y el aprendizaje, los cuales el docente debe aprender a afrontar y resolver. Entre estos, llama la atención el uso del tiempo escolar, como se muestra en la siguiente cita: no se pudo ejecutar debido a una actividad que se realizó en toda la escuela de un bingo para recoger fondos. Asimismo, en el área de matemática no se pudo trabajar el contenido estipulado para ese día, ya que en la escuela se realizo una actividad de un mega match con los niños y niñas de primera etapa y no se pudo reprogramar. [P5, 113:118].

Situaciones como estas son reiterativas en las aulas de clase: múltiples actividades que en algunos casos son descontextualizadas de las intencionalidades educativas, lo cual deriva en el desaprovechamiento del tiempo escolar y escasamente contribuyen a la formación de los niños. Incluso podría afirmarse que pueden generar sentimientos encontrados dado que se dispone de un tiempo escolar en el cual deben desarrollar tareas formativas, pero que, ante la demanda de actividades, ocasiona dudas y miedos. La siguiente cita lo explica: Me sentí con la mente en blanco, eran tantas cosas que debía hacer que no sabía cómo empezar el proyecto, que escribir en la planificación, no sabía cómo hacerlo eran demasiadas dudas juntas [P3, 59:62]. 
Asimismo, emergen emanadas del contexto emociones placenteras o no que, de algún modo, contribuyen a enriquecer la experiencia y orientan el aprendizaje de los futuros profesores, por ejemplo: Aparte del miedo que sentí sobre el trato de los estudiantes hacia mí como pasante [P3, 59:62]. Me sentí positivo ya que estaba seguro de lo que estaba haciendo, pienso que estos son retos que debemos enfrentar para poder fortalecer esos temores o debilidades que se nos presenta en nuestra primera práctica de docente [P8, 24:28].

Tal como se describe, esta categoría refiere algunos eventos que pudieran contribuir con la construcción del conocimiento docente, específicamente del pedagógico que parece tener relevancia y significado para los participantes por cuanto este conocimiento les permiten poner en acción la teoría construida durante el trayecto de formación. Shulman (2001) expresa que el conocimiento pedagógico general debe considerar "aquellos principios y estrategias generales de manejo y organización de la clase que trascienden el ámbito de la asignatura" (pág.174) de modo que según los eventos que reportan los estudiantes participantes en el estudio; este conocimiento forma parte fundamental del aprendizaje docente.

\section{Categoría: Experiencias formativas}

Tal como se muestra en la Figura 2, las Experiencias Formativas se hacen notables en tanto ofrecen la oportunidad de interactuar desde las prácticas con el contexto escolar y sus múltiples interrelaciones. Sin duda, se trata de oportunidades para comprender, interpretar y ejercer la docencia en un contexto real, lo cual permite que estas vivencias favorezcan la construcción de aprendizajes y el desarrollo de competencias para enseñar. Sobre esta base surgen las subcategorías Aprendizajes Construidos y Competencias.

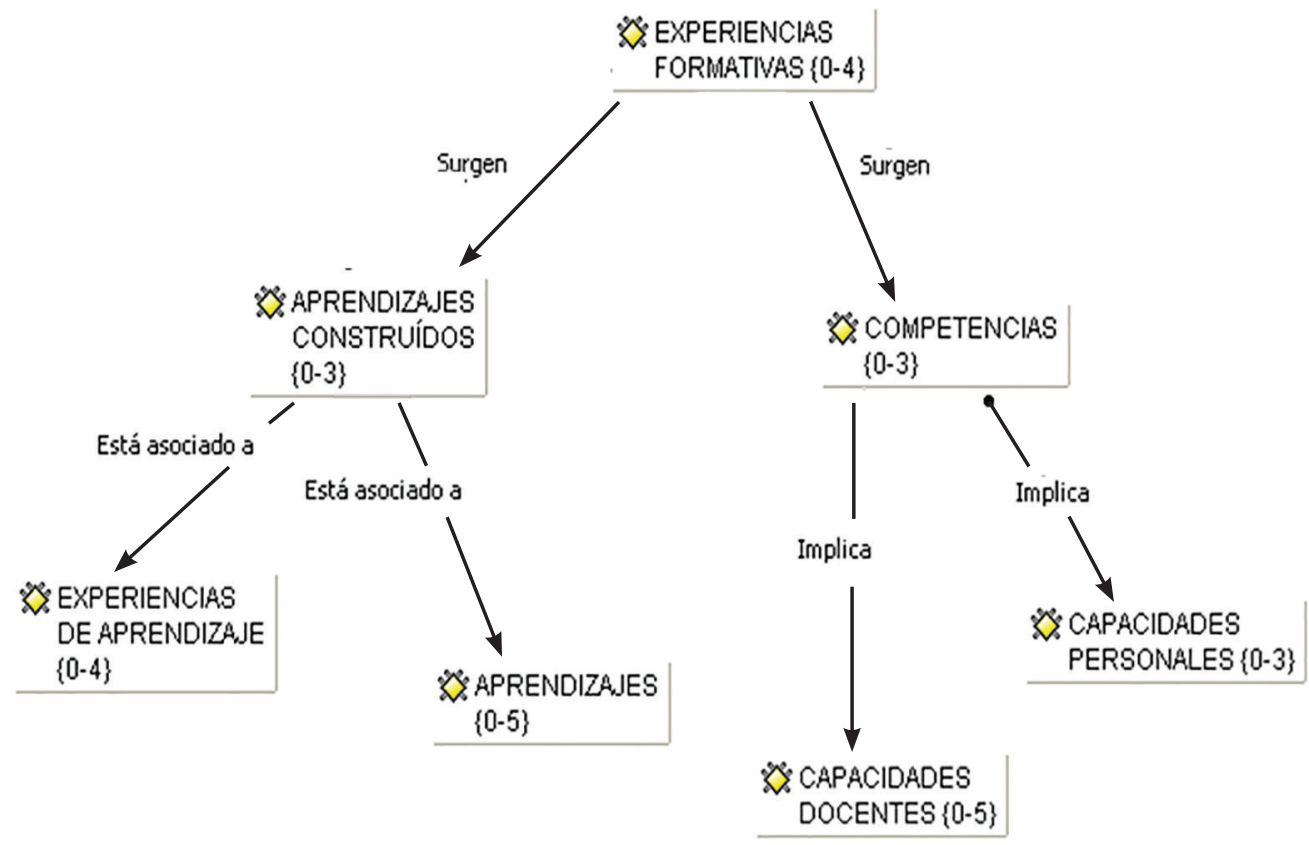

Figura 2. Categoría Experiencias formativas.

Nota: Proceso de investigación 
En relación con la subcategoría Aprendizajes Construidos, los participantes reconocen que ser docente constituye un aprendizaje permanente: el docente debe estar en constante capacitación y formación, pues a diario se necesita mucho más aprendizaje para así enseñar y educar [P6, 140:141]. Igualmente, comprenden que como docentes han de ser creativos e innovadores a fin de coadyuvar con el desarrollo integral de los escolares que tienen a su cargo, según afirma el participante siguiente: Aprendí que los niños y niñas que están frente a nosotros son exploradores innatos e inagotables por naturaleza, que no debemos cortar la imaginación de un niño por tonta que parezca, que de ellos también se aprende y que no son como loritos repitiendo y copiando cada modelo que está frente a ellos. Son seres con gran capacidad que debemos saber y como hacer brillar en todo su esplendor [P2, 56:61].

Otro aspecto que puede resaltarse es que se privilegia la práctica como un espacio para aprender a ser docente. Cabe destacar que la mayoría de estos participantes no había tenido una experiencia similar por tiempo prolongado en las aulas de clase. De allí que se trató de la primera vez que asumían un aula de clase como docentes. Un ejemplo se muestra seguidamente: he aprendido muchísimo, pues el ejercicio de la docencia me presentó en esta primera experiencia grandes retos, en especial el dominio de grupo, que debo hacer y no hacer para que los niños no se desordenen [P10, 44:46]. También algunos manifestaron contraste entre su rol como practicantes en los espacios escolares y la formación universitaria. La siguiente cita es una muestra: ir al aula de clase no es lo mismo a recibir las clases de la universidad ya que la realidad a la que te enfrentas es otra. Pero si es de gran ayuda en muchos momentos. Poco a poco uno se adapta [P15, 142:144].

Estos comentarios sugieren la percepción de los participantes sobre esta experiencia; sin embargo, también puede interpretarse como un alerta en tanto afirman, por una parte, que han aprendido; pero, por otra, también hablan de adaptación. Por tanto, surge la necesidad de que los centros educativos donde realicen la práctica les proporcionen experiencias significativas para su aprendizaje como docentes. Lo contrario hará que solo se "adapten" a la cultura institucional, la cual, en algunos casos, no es precisamente propiciadora de aprendizaje y trastocaría el carácter formativo de esta importante etapa de la formación inicial.

Asimismo se evidencia que la preocupación mayor de los pasantes radica en "controlar el grupo". Faltan mayores niveles de reflexión que orienten la acción en el aula hacia el uso de estrategias innovadoras que desencadenen aprendizajes interesantes tanto para el alumno como para el aprendiz de docente.

En cuanto a la subcategoría Competencias se refiere a aquellos conocimientos, habilidades, destrezas y capacidades que han venido desarrollando durante el trayecto formativo y en el período de prácticas; esto es, según Tobón (2006) "procesos complejos que las personas ponen en acción-actuación-creación para resolver problemas y realizar actividades" (pág.49). Se trata de movilizar el saber ser, saber conocer y saber hacer durante el desempeño profesional a fin de aportar soluciones y trasformar la realidad.

Con base en ello, los participantes expresan que ser docente implica: Capacitarse, prepararse, especializarse. Contar con un buen material de apoyo, actualizarse. Tener una buena actitud. No ser un maestro tradicionalista [P4, 152:154]. Siempre hay que prepararse, leer e investigar cada tema que se va a ejecutar, además de plantearse posibles preguntas 
y respuestas que suelen hacer los niños para saber abordarlas y si se desconoce el tema, incentivar la indagación para el aprendizaje de todos [P19, 76:79].

Estos testimonios podrían ser considerados importantes debido a que, para este grupo de participantes, las prácticas contribuyen en su formación profesional; por lo menos, constituyen un soporte para asumir el continuo desarrollo de competencias para desempeñarse como profesionales de la docencia. Tal como sostiene Pérez (2010) "los docentes han de formarse como investigadores de su propia práctica, para identificar y regular los recursos implícitos y explícitos que componen sus competencias y cualidades profesionales (p. 47).

Así, como se muestra en la Figura 3, la subcategoría competencia tiene asociada dimensiones vinculadas a las características personales, pero también a las capacidades docentes que corresponden a competencias profesionales que el futuro docente debe desarrollar para ejercer su labor profesional. A estas capacidades se refirieron los participantes como puede observarse en los siguientes testimonios: ser investigador y que siempre busque la excelencia en sus trabajos y actividades, crítico en sus juicios, humanista y con vocación de esta profesión en el alma, curioso ya que despierta la creatividad y la búsqueda de enseñar con innovación. Por otro lado, saber organizar, planificar, evaluar, es decir, todo lo que se pueda saber y lo que no se aprende para lograr el éxito en su profesión [P2, 93:98]. He aprendido que como docente debo ser un gran mediador, debo investigar muchísimo para prepararme en el día a día [P13, 30:31].

Las competencias profesionales abarcan capacidades cognitivas, sociales, y actitudinales que ponen en acción los docentes cuando asumen la labor de enseñantes. De acuerdo con quienes participaron en el estudio, los profesores deben desarrollar y movilizar competencias investigativas, de trabajo colaborativo, didácticas las cuales implican los saberes esenciales. Tobón (2010) expresa que "las competencias docentes son las que efectivamente se ponen en acción en las prácticas educativas cotidianas" (p. 273). Aunado a ello, un docente también debe saber cómo actuar ante situaciones de incertidumbre y contribuir con su desempeño en la resolución de problemas, lo cual implica saberes profesionales mucho más complejos que probablemente los pasantes aún están por descubrir.

\section{Categoría Competencia reflexiva}

Representada por las capacidades que los participantes han desarrollado para examinar y cuestionar sus propias acciones a fin de describir y confrontar su práctica pedagógica. En tal sentido, se preguntan o intentan justificar sus actuaciones y plantearse posibles soluciones. Durante estas deliberaciones surge la dicotomía entre teoría y práctica aunada al instrumentalismo. En detalle veremos cómo se evidencia la competencia reflexiva (ver Figura 3) a través de las subcategorías Postura Reflexiva y Reflexión en y sobre las Acciones.

La subcategoría Postura reflexiva se encuentra vinculada a la formación del habitus, es decir examinar nuestros esquemas, nuestras estructuras de percepción, evaluación y de acción (Bourdieu, citado por Perrenoud, 2004). En otras palabras, se trata de prepararse para dilucidar nuestros esquemas de pensamiento y de acción, lo cual posibilitaría formar el habitus al que se refieren los autores mencionados para concienciar y encontrar sentido a la manera cómo se asumen y resuelven determinadas situaciones, en las que se puede 
acudir o no a la formación académica. El detalle es ser capaz de explicarse determinados comportamientos y justificar las actuaciones.

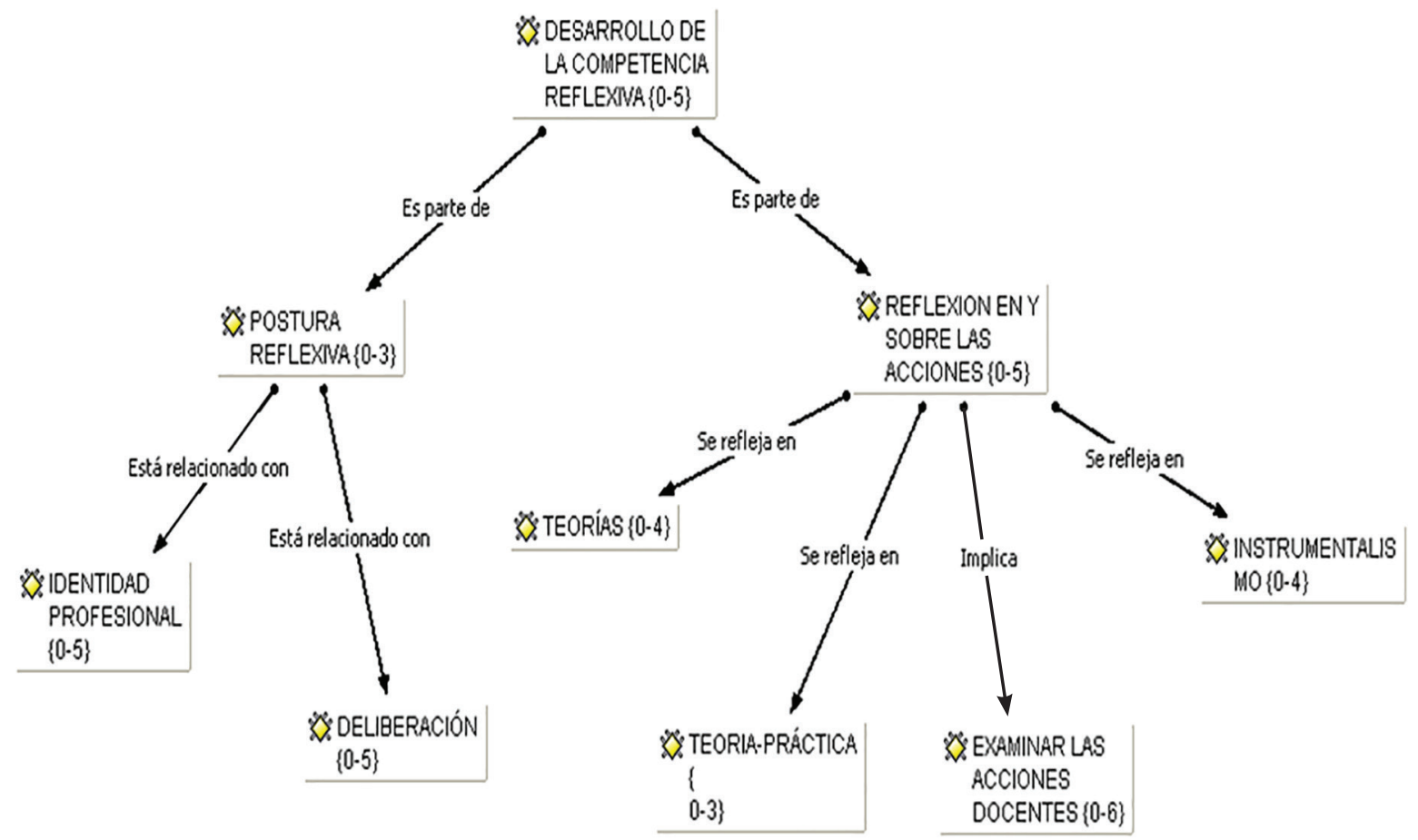

Figura 3. Categoría Competencia reflexiva.

Nota: Proceso de investigación

Lograrlo puede ser posible en tanto se forme reflexivamente a los futuros educadores. Se trata de asumir la complejidad de enseñar y estar consciente de que, a veces, se puede saber qué hacer ante determinadas situaciones y sin embargo, actuar contrariamente, incitados por el habitus. Por ello, la necesidad de explicitar nuestro saber hacer. De los testimonios a continuación, se desprende la necesidad de enfatizar en el desarrollo de una postura reflexiva que surge de manera incipiente aún, vinculada con algunos rasgos identitarios y la deliberación sobre la enseñanza:

El estar dentro del aula, y delante de un grupo de niños y niñas con la sed de aprender cada día más cosas y sobre todo de quien tienen en frente, hace que realmente se sienta la obligación de asumir la realidad de lo que significa el término "Enseñar", pues, es allí, donde se proyecta el trabajo que debo emprender. Estas prácticas, realmente llenaron en gran parte mis expectativas e inquietudes como: ¿será que puedo ser educador?, ¿realmente esta es la profesión que quiero ejercer durante mi existencia? ¿Podré enseñar algún día? y otras más. Sin duda alguna, hoy puedo decir que nada es fácil, pero tampoco difícil [P6, 55:63]. Solo es docente aquel que de su corazón nace la voluntad de servir y de enseñar a los demás un camino mejor con amor y sabiduría. [P2, 51:52]. Cada 
día es diferente al anterior y hay que estar preparados para afrontar las adversidades que se nos puedan presentar [P7, 24:25]. Para aprender me ha tocado arriesgarme, dar todo de mi, aplicar mis conocimientos y experiencias previas, así mismo equivocarme y mejorar aquellas observaciones que me hacen los docentes [P13, 43:45].

En cuanto a la subcategoría Reflexión en y sobre las Acciones Docentes, se encuentra la deliberación consciente de las acciones del aula. Los participantes enfrentados a la labor diaria se ven obligados a reflexionar sobre los obstáculos, situaciones y decisiones que han de tomar antes y durante la clase; es parte de actuar siguiendo las pautas de la planificación elaborada o, ante los imponderables que surgen en un contexto dinámico, multidimensional y complejo como el escolar. Así surgieron comentarios como:

Necesito mejorar en algunos aspectos como es la planificación en lo que se refiere a incluir actividades que sean más innovadoras y creativas para los niños, además de lo mencionado anteriormente en cuanto a la cantidad de los contenidos de la planificación. Además del dominio de grupo para tener un mayor control de aula, mostrando carácter ante ellos, sin caer tanto en los regaños y tener en cuenta que cada niño tiene un modo ser diferente y hay que saberlos tratar y en ocasiones, tenerles más paciencia a unos que a otros [P11, 72:79]. Necesito saber más en relación a las estrategias de aprendizaje existentes, a cómo mejorarlas e innovar otras más para mejorar la práctica en el aula y que los estudiantes de primaria logren obtener aprendizajes duraderos y de calidad [P15, 197:200].

De las citas anteriores se evidencia la necesidad de asumir la propia formación a fin de responder a las exigencias que marcan las características del contexto escolar. También puede señalarse que se trata de reflexiones sobre el saber hacer docente, es decir, la dinámica escolar desvela a los practicantes sus carencias en relación con las competencias profesionales, hecho que resulta interesante pues se hace inminente tomar la iniciativa para consolidarse como profesor.

Igualmente, emerge otro elemento importante en estos procesos reflexivos incipientes, pero de gran valor. Se trata de la sobrevaloración de la práctica que también surgió en estudios anteriores (Chacón, 2008). Una evidencia es el siguiente testimonio:

Por supuesto comprobé que la teoría pierde su esencia si no se lleva a la práctica, ésta por su parte, presenta obstáculos y no hay recetas para ser un docente bueno [P11, 48:51]. He aprendido mucho durante estas pasantías, puedo decir que lo que no pude aprender durante mi carrera lo aprendi en estas semanas de largo trabajo [P3, 42:44]. Ejerciendo mi rol como docente ha sido una experiencia extraordinaria. Tienen razón los docentes que me dieron clase durante la carrera cuando decían "la universidad solo te da un poco de teoría, pero la práctica la hacen ustedes mismos", o "la universidad te da el 10\% de lo que necesitas, el otro 90\% lo das tú"[P4, 45:49]. Mantener a los estudiantes ocupados ya que si no se tienen trabajando se desordenan fácilmente y luego es más difícil asignarles alguna tarea porque les parece tedioso por haber perdido el ritmo [P3, 181:183]. Comprendí la importancia de conocer los contenidos básicos de cada área, debido a que, ser docente integrador es un gran reto para los estudiantes de educación [P16, 61:63]. 
Las aseveraciones anteriores muestran la importancia que se otorga a las prácticas para aprender a ser docente, lo cual pudiera interpretarse como una sobrevaloración de la práctica, quizá producto de la escasa interacción con contextos escolares reales durante el trayecto de formación. Igualmente, se examinan los saberes de las disciplinas escolares en cuanto al saber y saber hacer de un docente y emerge, así, el instrumentalismo mediante la aplicación de técnicas o tareas. Pareciera que es imprescindible "mantener ocupados a los alumnos" para conservar el orden cuando el énfasis debe ser mediar aprendizajes significativos a través de estrategias de enseñanza innovadoras que activen procesos de pensamiento y planteen el análisis y resolución de problemas en los contextos escolares. Visto de este modo la práctica reflexiva es una alternativa posible, como sostiene Litwin (2008):

Construir y compartir espacios de reflexión posibilitan a los estudiantes y docentes enmarcar a la clase en un aura reflexiva, aventurarse en las hipótesis, prestar atención a las interpretaciones diferentes y cuestionar todas las acciones compartidas desde la perspectiva del conocer. (p.215)

En otras palabras, se trata de encontrar formas de enseñanza que propicien aprendizajes útiles tanto para el alumno como para el futuro profesor por cuanto aprender a enseñar trasciende lo meramente aplicativo; es comprender la profesión en un contexto determinado, atender las demandas sociales para educar en una sociedad más justa y solidaria.

\section{Discusión}

La construcción del conocimiento sobre la enseñanza es compleja, multidimensional y proviene de variadas fuentes, lo cual exige procesos reflexivos que den cuenta de los progresos y limitaciones, tanto en la formación inicial como permanente. En tal sentido, los aprendices de docencia comprenden la necesidad de conocer las disciplinas que enseñan y el contexto escolar. Asimismo, otorgan un lugar preponderante al conocimiento pedagógico que se traduce en la organización y gestión de la clase a través de la planificación y la evaluación. Del mismo modo, expresan interés por saber y aplicar estrategias de enseñanza que potencien los aprendizajes.

Por otra parte, privilegian las prácticas profesionales como un ámbito importante de la formación y expresan que durante el trayecto formativo tuvieron escasas posibilidades de interactuar en los contextos escolares. Es preciso afirmar que estos espacios ofrecen la oportunidad de explicitar un conjunto de conocimientos complejos que se ponen en acción durante la enseñanza. Esta interacción contribuye a construir el saber práctico, que algunos autores como Korthagen (2010) y Zeichner (2010) han caracterizado como aquel que proviene de las experiencias de los profesores, comprende las actitudes, valores, creencias personales; así como el conocimiento que se posea del contexto donde se interviene: escuela-aula. Entonces, el aula es un espacio donde, según explica Díaz (2009), "se articulan los conceptos teóricos estructurados" (p.127), lo que posibilita la recuperación de la concreción de la enseñanza a partir de la relación teoría práctica, haciéndose evidente la construcción del conocimiento docente.

A pesar de las consideraciones anteriores, todavía persiste la desconexión entre teoría y práctica en el trayecto formativo. De hecho, algunos de los participantes perciben que en la universidad no aprenden lo suficiente para interactuar en las instituciones educativas. 
Esta realidad, exige la revisión de las prácticas de los formadores de docentes; en tanto es necesario mediar entre los aprendizajes y las experiencias formativas, de manera que los futuros profesores puedan elaborar las necesarias conexiones entre teoría práctica.

Al respecto, Zeichner (2010) agrega que "el problema perenne de los programas de formación del profesorado en las universidades tradicionales ha sido la falta de conexión entre la formación ofrecida en el campus universitario, las asignaturas y las experiencias de la práctica" (p.128), si bien, reconoce el mismo autor, se hacen esfuerzos por establecer puentes para proveer oportunidades de aprendizajes significativos, todavía existe una brecha que es necesario superar. En otras palabras, se mantiene la desconexión entre la formación en las aulas universitarias y la realidad escolar, de manera que el reto es establecer los vínculos necesarios para superar la dicotomía teoría práctica.

Así pues, emerge el desarrollo de procesos reflexivos a propósito de analizar las acciones docentes, repensar acerca de las concepciones y núcleos temáticos vinculados a los procesos formativos. Esto supone examinar las creencias y el habitus que poseen los futuros profesores, al tiempo que conocen la institución escolar, sus prácticas y su cultura. Todo ello debido a que, gran parte de su vida, han convivido en las aulas escolares y cuando regresan como docentes, de algún modo, rescatan sus vivencias, maneras de enseñar y aprender de las cuáles fue objeto.

Korthagen (2010) es partidario de impulsar la reflexión para concienciar sobre la enseñanza y sus implicaciones con base en sus propias experiencias, pero esta reflexión debe ser sistemática, explícita y debería conducir hacia la autonomía e impulsar el aprendizaje reflexivo entre pares. De ahí la importancia de la construcción de comunidades de práctica. Uno de los aportes de este estudio, de acuerdo con los hallazgos, es que reflexionar exige disposición a aprender en contextos reales de aprendizaje. Por tanto, la reflexión orientada hacia el desarrollo de estrategias de aprendizaje puede coadyuvar en la construcción del conocimiento docente, siempre que se enfatice en la enseñanza reflexiva como posibilidad para aprender acerca de la enseñanza.

Es prioritario dirigir la formación inicial hacia el desarrollo de una práctica reflexiva como el centro de la formación docente, lo cual significa que los formadores de formadores deben adscribirse, de hecho, como profesionales reflexivos porque la reflexión no se trasmite tácitamente, sino que implica poner en marcha una serie de estrategias de formación con el propósito de desarrollar la competencia reflexiva. Estas acciones incluyen el análisis de la práctica de cara a los postulados teóricos, didácticos, pedagógicos imbricados en la acción docente. Aunado a ello, el trabajo colaborativo, los seminarios de prácticas, entre otras estrategias que no son aisladas, ni individuales sino que posibilitan la integración de éstas con las contextos escolares fin de reconstruir el conocimiento de manera colectiva y cooperativa. Solo queda reiterar que la formación de profesionales reflexivos sigue siendo un reto que la universidad, como institución formadora, no acaba de asumir.

\section{Referencias}

Badía, A. y Monereo, C. (2004). La construcción de conocimiento profesional docente. Análisis de un curso de formación sobre la enseñanza estratégica, Anuario de Psicología 35 (1), 47-70. Recuperado de http://dialnet.unirioja.es/servlet/articulo?codigo=864598 
Bromberg, A., Kirsanov, E. y Longueria, M. (2008). Formación profesional docente: Nuevos enfoques. (2 ed). Buenos Aires, Argentina: Bonum.

Coffey,A.y Atkinson,P.(2003). Encontrar sentidoalosdatos cualitativos. Estrategiascomplementarias de investigación. Colombia: Contus Editorial de la Universidad de Antioquia.

Chacón, M. (2008). Las estrategias de enseñanza reflexiva en la formación inicial docente. Educere, 12(41), 277-287. Recuperado de http://www.redalyc.org/articulo.oa?id=35611336007

Cruz, M, Pozo, J., Huarte, M. y Scheuer, N. (2006). Concepciones de enseñanza y prácticas discursivas en la formación de profesores. En J. Pozo, N. Scheuer, M. Pérez, M. Mateos, E. Marín y M. Cruz (Dirs), Nuevas formas de pensar la enseñanza y el aprendizaje. Las concepciones de profesores y alumnos. pp. 359-371. Barcelona, España: Graó.

Davini, M. (2001). La formación docente en cuestión: Política y Pedagogía. Argentina: Paidós.

Díaz, A. (2009). Pensar la didáctica. Buenos Aires, Argentina: Amorrortu.

Diker, G. y Terigi, F. (2003). La formación de maestros y profesores: hoja de ruta. Buenos Aires, Argentina: Paidós.

Elliott, J. (1999) La relación entre comprender y desarrollar el pensamiento de los docentes. En Desarrollo profesional docente: Política, investigación y Práctica. En J. Angulo, J. Barquín y A. Pérez (Eds.). pp. 364-398. Madrid, España: Akal.

Korthagen, F. (2010). La práctica, la teoría y la persona en la formación del profesorado. Revista Interuniversitaria de Formación del Profesorado, 68, 83-101.

Litwin, E. (2008) El oficio de enseñar. Condiciones y Contextos. Buenos Aires, Argentina: Paidós.

Martín, E. y Cervi, J. (2006). Modelo de formación docente para el cambio de concepciones en los profesores. J. Pozo, N. Scheuer, M. Pérez, M. Mateos, E. Marín y M. Cruz (Directores). Nuevas formas de pensar la enseñanza y el aprendizaje. Las concepciones de profesores y alumnos. pp. 419-434. Barcelona, España: Graó.

Montero, L. (2002). La formación inicial, ¿puerta de entrada al desarrollo profesional? Educar, 30, 68-89. Recuperado de http://mail.quadernsdigitals.net/datos_web/hemeroteca/r_73/nr_785/a_10661/10661.pdf

Pérez, A. (2010). Aprender a educar. Nuevos desafíos para la formación de docentes. Revista Interuniversitaria de Formación del Profesorado, 68, 37-60. 
Porlán, R., Rivero, A. y Martín, R. (1997) Conocimiento profesional y epistemología de los profesores I: Teorías, métodos e instrumentos. Enseñanza de las ciencias, 15 (2), 155-177.

Perrenoud, P. (2004). Desarrollar la práctica reflexiva. Barcelona, España: Graó.

Sayago, Z. y Chacón, M. (enero-marzo, 2006). Las prácticas profesionales en la formación docente: hacia un nuevo diario de ruta. Educere 10 (32), 55-66. Recuperado de www. redalyc.org/articulo.oa?id=35603209

Strauss, A. y Corbin, J. (2002). Bases de la investigación cualitativa. Técnicas y procedimientos para desarrollar la teoría fundamentada. Colombia: Contus Editorial de la Universidad de Antioquia.

Shulman, L. (2001). Conocimiento y Enseñanza. Estudios Públicos, 83, 163-196. Recuperado de http://132.248.9.34/hevila/EstudiospublicosSantiago/2001/no83/2.pdf

Shulman, L. (2005). Conocimiento y Enseñanza: Fundamentos de la Nueva Reforma. Revista de currículum y formación del profesorado, 9 (2), 1-30. Recuperado de http://www.ugr. es/ recfpro/rev92ART1.pdf

Tobón, S. (2006). Formación basada en competencias. Pensamiento complejo, diseño curricular $y$ didáctica. Bogotá, Colombia: Ecoe ediciones.

Tobón, S. (2010). Formación integral y competencias. Pensamiento complejo, currículo, didáctica y evaluación. (3 ed). Bogotá, Colombia: Ecoe Ediciones.

Tardif, M. (2004). Los saberes del docente y su desarrollo profesional. Madrid, España: Narcea ediciones.

Vaillant, D. y Marcelo, C. (2001) Las tareas del formador. Málaga, España: Ediciones Aljibe.

Zeichner, K. (Agosto, 2010). Nuevas epistemologías en formación del profesorado. Repensando la conexiones entre las asignaturas del campus y las experiencias de prácticas en la formación del profesorado en la universidad. Revista Interuniversitaria de Formación del Profesorado 24(2), 123-149. 